\title{
Symmetries in the Mathematical and Physical Description of Nature
}

\author{
Dr. Andreas Gimsa
}

Stirling Technologie Institut gemeinnützige GmbH, D-14478 Potsdam, Germany

\begin{abstract}
Symmetries play an essential role in nature. Symmetrical structures are generally perceived as beautiful Mathematicians and also physicists even regard symmetries in the equations for the mathematical and physical description of the world as an indication of their correctness. The British mathematician Godfrey Harold Hardy [1.] writes: "The mathematician's patterns, like the painter's or the poet's must be beautiful; the ideas like the colours or the words, must fit together in a harmonious way. Beauty is the first test: there is no permanent place in the world for ugly mathematics." A very interesting example of symmetries in physics has been provided by Emmy Noether, who found that certain system characteristics are preserved during changes (transformations). Emmy Noether derived the propositions of conservation of energy, momentum and angular momentum from the invariance (immutability) of the laws of nature during transformation of time, place and direction. These symmetries and their conservation laws form the foundation of physics. In this publication, further essential symmetries are to be investigated, which relate in particular to the symmetry of energy and information and their effects.
\end{abstract}

Keywords: Energy conservation, Information conservation, Magnetic monopoles, Neutrinos, Mass decay, Gravitation, Entropy, Planck units.

\section{Introduction}

The physical description of nature stands at a new threshold of knowledge. So the unification of gravitation and quantum physics must succeed. Time must not be taken for granted, it must be physically explained. The expansion of space and the fast rotation of stars in the outer regions of galaxies require a plausible explanation. Symmetries in nature will help to understand these natural processes. In order not to go beyond the scope of this publication and to present the symmetries in a clear way, some references are made to the sources for their explanation.

\section{Terms and definitions of important basic variables}

In the past, some physicists have given different definitions for the terms mass, energy and information. Other definitions outside of physics, often have a philosophical character. A useful definition of time has not yet been given. In this publication the mathematical structure of the terms is in the foreground [2.], [3.], [4.].

Mass $m \quad$ A certain number of structural elements is understood as mass. The greater their number, the greater the mass. It can also be understood as the inertia of matter.

Time $t \quad$ A certain number of structural changes is understood as time. The greater their number, the more time has passed or will pass. It can also be seen as the ability of matter to change.

Energy $E \quad$ Energy at rest state of the mass results from the change in mass and the change in shape, namely from the product of these two properties [5.].

$E=-\frac{d m}{d t} \frac{d A}{d t}$

This hitherto unknown but very essential definition is important for understanding universal mass decay, for linking classical physics and quantum physics, for explaining the constant entropy of space and for explaining gravity. 
Information $I$ Information is the structural change itself. This explains the concept of information in the simplest way and defines it independently of the sender and receiver of information. The following new formula also shows with (01) that information is a component of energy [6.]:

$$
I=\frac{d A}{d t}
$$

Matter It consists of mass and structure. It is temporal and therefore changeable.

Space Space is a changeable 3-dimensional structure. It is able to store negative energy (gravitational energy) and serves as a location and reference system for matter.

\section{Symmetry of space, real and imaginary time, and of light and imaginary light}

The connection and the symmetry between space and time can be explained best by the new 5-dimensional space-time equation, which was found by me [7.]:

$$
d x^{2}+d y^{2}+d z^{2}=c^{2} d t^{2}-c_{i}^{2} d t_{i}^{2}
$$

While the space dimensions $x, y, z$ and the time dimension $t$ are well known, the imaginary speed of light $c_{i}$ and the imaginary time $t_{i}$ require further explanation. The simple formula applies to the imaginary speed of light:

$$
c_{i}^{2}=-c^{2}
$$

It can be used to explain instantaneous processes, such as those occurring during the change of state of entangled quanta that are far away from each other. In addition, the imaginary speed of light opens up the possibility of instantaneous transfer of gravitation, as demanded by Newton (see also Laplace with his approach to transfer at superlight speed). The author has succeeded in deriving gravity from quantum physics. This derivation leads with transfer of the gravity with an imaginary speed of light on the Newtonian law of gravity [8.]. The imaginary photons were identified as gravitational interaction particles. It is assumed here that the virtual photons used to describe some quantum processes are also imaginary photons. These do not exist in the known time, but only in the imaginary time, which will be explained later. What does the imaginary time $t_{i}$ express?

The space age must not know a preferred place. With the definition of absolute simultaneity in the form of space age, the second temporal dimension appears as an imaginary dimension with past, present and future. The distinction between past, present and future in the imaginary dimension shall be explained:

1. A second event takes place in the imaginary present $t_{i g}$ to a first event here and now, if it takes place in the same space age at a different place, exact to the femtosecond.

2. A second event takes place in the imaginary past $t_{i v}$ to a first event here and now, when it is so far away that the time required for the light to travel from the second to the first event is not sufficient to exert a causal influence on the first.

For example, a solar eruption 6 minutes ago cannot influence the weather here and now. It lies in the imaginary past of the event here and now. The interesting thing is that this solar eruption can influence the weather here at a later time.

3. a second event lies in the imaginary future $t_{i z}$ to a first event here and now, when it is so far away that the time it takes the light from the first to the second event is not sufficient to exert a causal influence on the second. For example, a solar eruption in 6 minutes cannot be influenced by a laser beam emitted here and now in the direction of the sun. The event on the sun lies in the imaginary future of this laser beam emission. In 10 minutes another event on the sun could in principle be influenced by this laser beam.

Logic dictates that events that lie in the imaginary past or future of an event here and now and are acausal to that event can be causal to events closer elsewhere. Thus the definition of imaginary time in relation to real time is location-dependent.

Furthermore, as shown in chapter 5, the imaginary time $t_{i}$ can be used to describe the baryonic $m_{b}$ and the dark mass $m_{d}$. With the imaginary time there is therefore the possibility to introduce the mass into the space-time equation (03). 


\section{Symmetry of time}

From the considerations of the preceding chapter, the temporal symmetry of the two temporal dimensions $t$ and $t_{i}$ for the past and the future follows directly. Both dimensions appear both in a complex past and in a complex future. As usual for complex numbers, the representation takes place in normal form and in Eulerian exponential form. As shown above, each event here and now can be assigned a total past $t_{\text {vges }}$ consisting of real and imaginary past. Each event here and now can also be assigned a total future $t_{z g e s}$ consisting of real and imaginary future. The negative sign of the future is explained in chapter 5 .

3.1 Past

$$
t_{v g e s}=t_{v}+t_{i v} \quad t_{v g e s}=\left|t_{v}\right| e^{i \varphi}
$$

\subsection{Future}

$$
t_{z g e s}=-t_{z}-t_{i z} \quad t_{z g e s}=\left|t_{z}\right| e^{-i \varphi}
$$

With the help of these relationships, any event can be described in relation to other events in the space-time structure of space [9.]. With the imaginary presence $t_{i g}$, simultaneous events can be defined at arbitrary locations with respect to space age.

The imaginary time $t_{i}$ has a special property. Its square corresponds to the negative square of the real time $t_{b}$ in the moving state with the velocity $\mathrm{v}$ of an object compared to its resting state. Why this is so, was explained in source [10.]. It applies:

$$
-t_{i}^{2}=t_{b}^{2}=t^{2}\left(1-\frac{v^{2}}{c^{2}}\right)
$$

If one inserts this expression and the definition according to (04) into the integrated space-time equation (03), one arrives at a known relationship, such as that which applies to the speed of a particle in a gas container:

$$
x^{2}+y^{2}+z^{2}=v^{2} t^{2} \quad v=\sqrt{v_{x}^{2}+v_{y}^{2}+v_{z}^{2}}
$$

\section{Symmetry of the magnetic monopoles and the electron neutrino}

There are not many sources that put the elementary magnetic charge $\mathrm{p}$ into a formulaic context. In "Gerthsen Physics" the following relationship was given for the force ratio of two magnetic and two electric elementary charges [11.]:

$$
\frac{F_{m g}}{F_{e l}}=\frac{p^{2} \mu_{0} \varepsilon_{0}}{e^{2}}=\frac{1}{\alpha^{2}}
$$

This would mean that two magnetic elementary charges interact much more strongly (factor of approx. $20,000)$ than two electric ones.

On the other hand, if you divide a magnetic force with the magnetic elementary charge $\mathrm{p}$ by an electric force in a symmetrical structure, you get the following formula instead of (09):

$$
\frac{F_{m g}}{F_{e l}}=\frac{\frac{p^{2}}{4 \pi \mu_{0} r^{2}}}{\frac{e^{2}}{4 \pi \varepsilon_{0} r^{2}}}=\frac{p^{2} \varepsilon_{0}}{e^{2} \mu_{0}}
$$

Since equations (09) and (10) are contradictory, they will be examined. First the SI units are analysed. With the well-known formula $1 / c=\sqrt{\varepsilon_{0} \mu_{0}}$ and (09) the following would simply apply: $p=e c / \alpha$ ? The monopoly $p$ would have to be assigned the unit [Am] because $e$ has the unit [As].

According to equation (10), however, $\varepsilon_{0}$ in $[\mathrm{As} / \mathrm{Vm}]$ and $\mu_{0}$ in $[\mathrm{Vs} / \mathrm{Am}]$ would result in the unit [Vs] for $\mathrm{p}$. This seems plausible. The further investigation includes the electric and magnetic field strength. At an electron that experiences a force $\vec{F}$, the electric field strength $\vec{E}=\vec{F} / e$ prevails. The electric field strength is therefore in the unit $[\mathrm{V} / \mathrm{m}]$. Now there should be an analogy according to which a magnetic field strength $\vec{H}=\vec{F} / p$ prevails at a monopole which also experiences a force $\vec{F}$. The magnetic field strength has the unit $[\mathrm{A} / \mathrm{m}]$. However, this unit can only be obtained by using the unit [Vs] from (10) for $p$. If the unit [Am] from 
(09) is used for $p$, a unit of the magnetic field strength of [ $\left.\mathrm{Vs} / \mathrm{m}^{2}\right]$ is obtained. However, this is the unit of magnetic flux density $\vec{B}$.

It shall be investigated how large the force ratio $F_{m g} / F_{e l}$ actually is. For this purpose, the following experimental arrangement in a vacuum is proposed. It can only be considered from a theoretical point of view, since magnetic monopoles are not accessible to us as time formers in space. This is explained later in this chapter [12.].

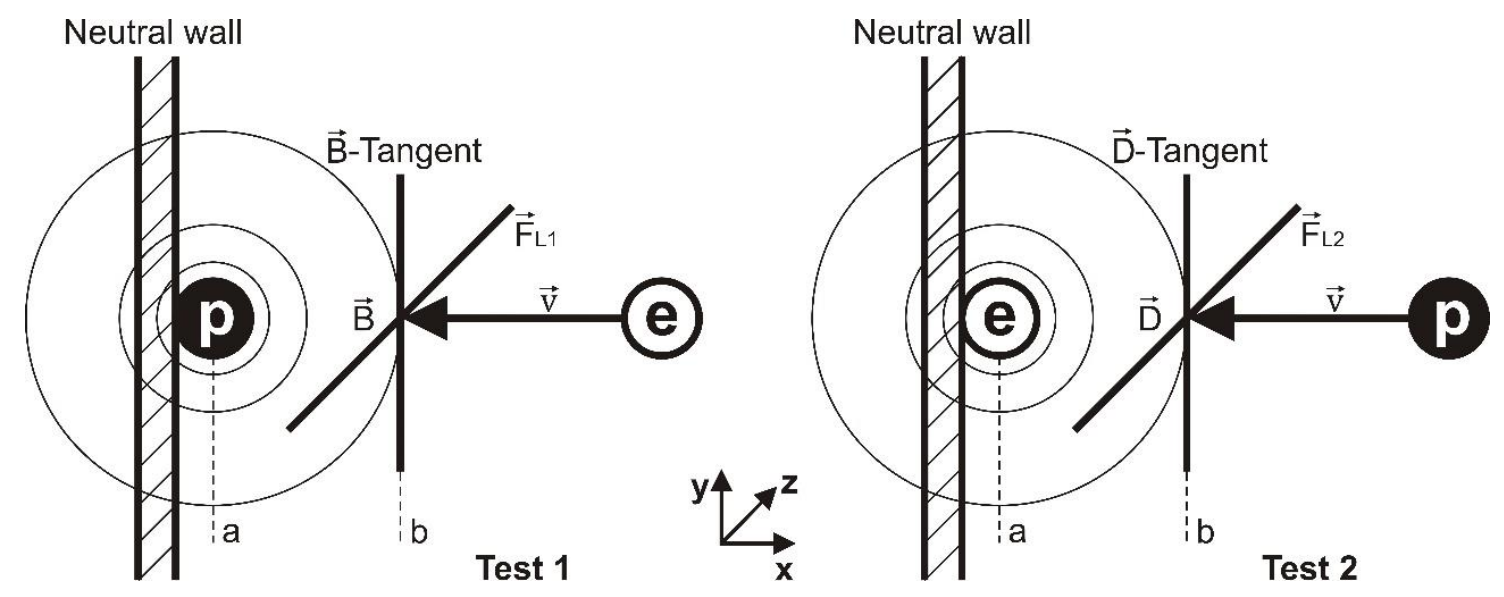

Figure 1: Symmetrical effect of the Lorentz force

The first experiment envisages that an electric elementary charge $e$ flies in the direction of a magnetic elementary charge $p$ attached to an electrically and magnetically neutral wall at the velocity $\vec{v}$ through the space point on the b-line. This means that there is no external electric field for the electric charge $(\vec{E}=0)$, only that of the magnetic elementary charge. The Lorentz force with a moving electric elementary charge is then at this point in space:

$$
\vec{F}_{L 1}=e \vec{E}+e(\vec{v} \times \vec{B})=e(\vec{v} \times \vec{B})
$$

In the second experimental arrangement, the electric elementary charge $e$ is now attached to the wall and the magnetic elementary charge $p$ flies at the same speed $\vec{v}$ through the point in space at the b-line towards $e$. There is no external magnetic field for the magnetic elementary charge $(\vec{H}=0)$, only the electric field of the elementary charge $e$. Since this experimental arrangement is symmetrical to the first, the following formulation must apply here, with the moving magnetic elementary charge at the same point in space:

$$
\vec{F}_{L 2}=p \vec{H}+p(\vec{v} \times \vec{D})=p(\vec{v} \times \vec{D})
$$

If point charges are assumed and the direction of movement of the charges on the respective field lines is vertical, equations (11) and (12) are simplified in such a way that the vector products are omitted and all vectors are reduced to their amounts:

$$
\begin{aligned}
& F_{L 1}=e v B \\
& F_{L 2}=p v D
\end{aligned}
$$

Since both attempts are exactly the same problem, the following applies:

$$
F_{L 1}=F_{L 2}=e v B=e v \mu_{0} H=p v D=p v \varepsilon_{0} E
$$

From this, the ratio of electric and magnetic field strength can now be determined as the wave resistance of the wave propagating in a vacuum:

$$
\frac{E}{H}=\frac{e \mu_{0}}{p \varepsilon_{0}}=Z_{0}=\sqrt{\frac{\mu_{0}}{\varepsilon_{0}}} \text { oder } \frac{p \varepsilon_{0}}{\mathrm{e} \mu_{0}}=\sqrt{\frac{\varepsilon_{0}}{\mu_{0}}}
$$

Equation (16) in turn can be converted into the ratio of magnetic to electrical force. As expected, the calculation formula is in accordance with equation (10).

$$
\frac{F_{m g}}{F_{e l}}=\frac{p^{2} \varepsilon_{0}}{e^{2} \mu_{0}}=1
$$


The force between two magnetic monopoles is as great as the force between two elementary electric charges. The equation (09) is wrong when considered in SI units.

For the wave resistance of the vacuum, equation (16) can be written very easily:

$$
Z_{0}=\frac{p}{e}
$$

Using the well-known equation (19), it is now possible to easily give a comparable formula for the magnetic elementary charge $p$ :

$$
\begin{aligned}
& e^{2}=\alpha 4 \pi c \varepsilon_{0} \hbar=2 \alpha c \varepsilon_{0} h \\
& p^{2}=\alpha 4 \pi c \mu_{0} \hbar=2 \alpha c \mu_{0} h
\end{aligned}
$$

If you multiply equation (19) by (20) you get the following concise expression after simplification:

$$
h=\frac{1}{2 \alpha} e p
$$

Now the Klitzing-resistor $R_{K l}=h / e^{2}$ with equation (21) can get the following form:

$$
R_{K l}=\frac{1}{2 \alpha} \frac{p}{e}
$$

It is known that the energy of an oscillating system is quantized via Planck's quantum of action $h$ with certain energy amounts at its frequency. Dirac has predicted for the magnetic charge a quantization with integer multiples of $e / 2 \alpha$ and a force action between two monopoles, which is $1 / 4 \alpha^{2}=4,691$ times as strong as that between two electrons [13.]. The energy quantum condition is applied to equation (21): $N e p / 2 \alpha$. The quantum condition for the Hall resistance over the Klitzing resistance according to equation (22) is: $p / N 2 \alpha e$. If you divide the first quantum condition by the second quantum condition, you immediately arrive at the necessary quantisation of the electric charge: From $N^{2} e^{2}$ follow integer multiples of e for each electric charge: $Q_{e}=N e$. Multiplying both quantum conditions leads to the expression $p^{2} / 4 \alpha^{2}$. This means that the magnetic charge is quantised with integer multiples of $p / 2 \alpha: Q_{m}=N p / 2 \alpha$. The force between the smallest magnetic charges $Q_{m}$ must therefore be 4,691 times greater than that between the smallest electric charges $Q_{e}$.

The elementary magnetic charge $p$ can now be easily calculated using equation (21):

$$
p=6.03610^{-17} \mathrm{Vs}
$$

An electron neutrino could consist of two magnetic monopoles. Neutrinos are uncharged neutral particles. The neutrality of a massy particle can be explained from its inner structure, only with a compensating charge ratio. This means that every neutrino or antineutrino would have to consist of at least one pair of opposite charges.

Because the electron neutrinos are the particles with the smallest known mass and their neutrality is chargerelated, it is therefore assumed that they consist of two oppositely charged magnetic monopoles, a magnetic north pole and a magnetic south pole. This neutrino would then be a magnetic dipole, whose poles may have been intertwined before decay. This could explain the external neutrality. Although it is difficult to test these considerations experimentally, it is physically untenable to simply take time as a given. 


\section{The origin of time and the symmetry of mass in the universe}

The neutrino decay

The real part of the future

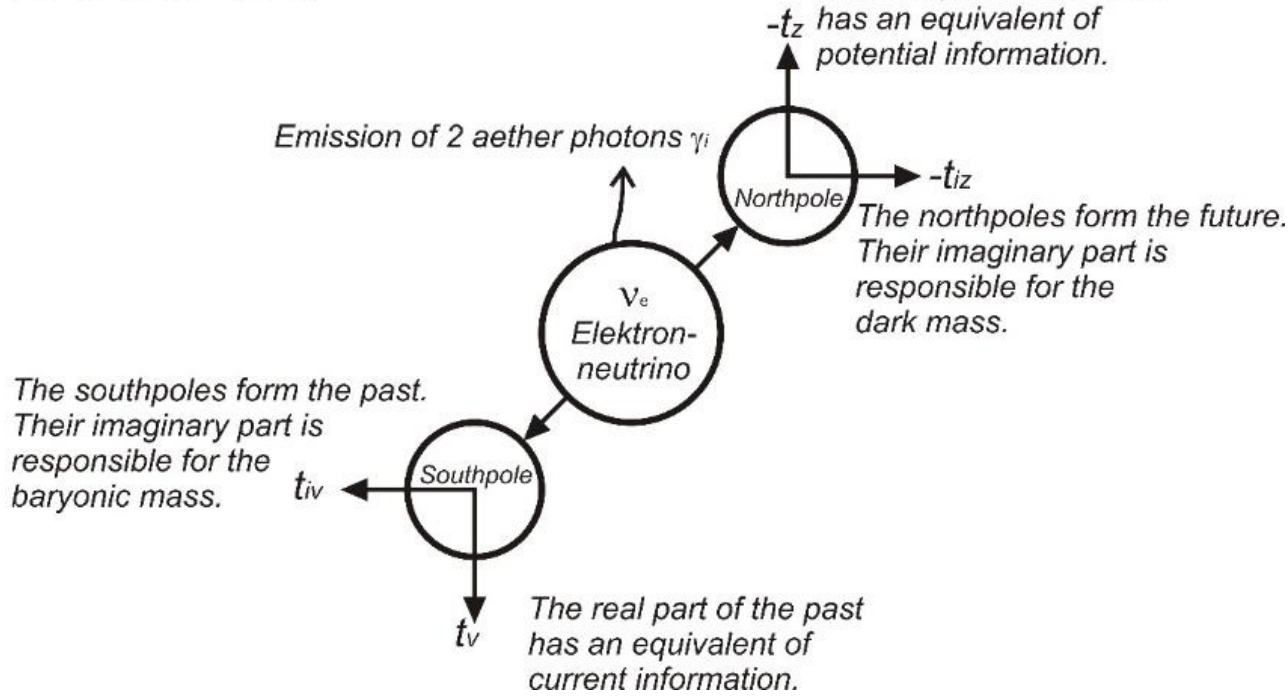

Figure 2: Mechanism of time formation from the decay of electron neutrinos

Figure 2 shows the presumed electron-neutrino decay, which produces a magnetic north pole and a magnetic south pole. Both monopoles form the complex time and each has a real and an imaginary part. The south pole should be the antiparticle of the north pole. It is repelled by the north pole. If this were not the case, both quanta in the neutrino would annihilate each other when energy is released.

Can charges of the same polarity attract each other and repel charges of different poles? It is already known that normal masses attract each other with the same magnetic polarity. What is not known is that oppositely poled masses repel each other. Common processes, such as the electrical attraction between a positive and a negative particle, take time. However, if it is a process in which time is created, the process should appear backwards in time. In this process, charges of the same polarity attract each other and charges of different polarity repel each other. The forces of magnetic monopoles are strongly weakened in space, because they cannot exist here and fill the two time dimensions.

In space there are two types of masses, baryonic and dark mass. The baryonic mass has a positive sign and the dark mass should have a negative sign. So dark mass exists in the outer regions of the galaxy, attracts as negative mass of the same polarity among each other and pushes the stars with positive mass inwards on their orbits. For stable orbits, these stars must therefore have higher orbital speeds than expected [14.].

Between the baryonic masses and their two existing gravitational fields: 1 . in the region of the baryonic masses and 2. in the region of the dark masses, there must therefore be a magnetic potential $\varphi_{m}=\frac{Q_{m}}{4 \pi \mu_{0} r}$ [A] with the charge $Q_{m}[\mathrm{Vs}]$ at a distance $r$ [m]. This potential strives for an equalisation. This leads to a magnetic current $\vec{I}_{m}$ [V], which brings about charge equalisation. The change of the magnetic charge $Q_{m}$ with this current $\vec{I}_{m}$ is, in accordance with the then complete Maxwell equations, the time $t$ [s], which appears as time flow $t=d Q_{m} / d \vec{I}_{m}$.

Magnetic fields therefore have no sources or sinks in space. These are located in the two time dimensions, which are offset by $90^{\circ}$ to each spatial dimension. The south poles of the past come out of the masses after neutrino decay into the gravitational field near the masses. The north poles of the future arrive in the area of the dark masses of the gravitational field outside the galaxies (see also Figure 2).

The second and the third Maxwell equation can be extended as follows:

- The source of the magnetic field are charges and the magnetic field strength must be proportional to the charge density: $\operatorname{div} \vec{H}=-\rho_{m} / \mu_{0}$

- The temporal change of the magnetic field can cause an electric vortex field. However, this can also be caused by a magnetic current with a certain density: $\operatorname{rot} \vec{E}=-\vec{\jmath}_{m}-\mu_{0} \partial \vec{H} / \partial t$. This could explain the cause of the electron movements around the atomic nucleus, because in the case of mass decay the 
magnetic current $\vec{I}_{m}$ must flow through the masses. The rotational movements of celestial bodies can also be caused by the magnetic current.

The exchange particles between the magnetic charges of objects and fields are the imaginary photons $\gamma_{i}$ of velocity $c_{i}$. They could cause instantaneous interactions. According to my considerations, these phenomena include gravitation in addition to the meanwhile experimentally secured state transfer of entangled quanta.

Everywhere in space and in the masses time-forming neutrinos decay.

Space is in the expansion phase. In my opinion, there has not been an inflationary phase. It originated in the fantasy of physicists to justify the Big Bang. It is very probable that space will undergo a compression phase after expansion, during which the magnetic monopoles will fuse back together. The former future, which has a very small amount at the end of the expansion, becomes the past. Its amount grows again, while the former past becomes the future and then becomes smaller. Before that a reversal of all masses will take place. Instead of neutrinos, there will be antineutrinos in the compression of the universe, which have a small negative mass and which, like the neutrinos before them, attract each other. Although these symmetry properties are speculative, they are nevertheless captivating in their beauty.

Figure 2 shows that the imaginary past stands for the baryonic mass and the imaginary future for the dark mass. For the baryonic mass it is possible to write with the imaginary past and the imaginary speed of light:

$$
m_{\text {buni }}=\frac{t_{\text {iv uni }} c_{i}{ }^{3}}{\gamma}=\frac{t_{v \text { uni }} c^{3}}{\gamma}
$$

Now, however, the general mass decay of the universe with age requires a mass decrease. This seems to contradict equation (24). However, one must note that the gravitational value $\gamma$ must increase quadratically with age [15.].

If the baryonic mass and the past are positive, this does not apply to the dark mass and the future. A positive period in the past assumes a greater age today compared to an earlier time.

A future period which assumes a smaller age today than at a later time is therefore negative.

For the dark mass of the universe $m_{d \text { uni }}$, the future $t_{z \text { uni }}$ is related to the baryonic mass $m_{b \text { uni }}$ and the past $t_{v \text { uni }}$ :

$$
\left(-m_{\text {duni }}\right)\left(-t_{\text {zuni }}\right)=m_{d \text { uni }} t_{z \text { uni }}=m_{\text {buni }} t_{v \text { uni }}
$$

The negative sign of the future can also be explained by the structural information of the matter during its mass decay, because the gravitational energy released is also negative. The following consideration is interesting: If the dark mass is about 5 times as large as the baryonic mass, the future of the universe during the expansion phase, in which the mass falls, is about 0.2 times as large as the past with 13.8 billion years. The expansion of the universe will therefore continue for another 2.8 billion years! Then the difficult question remains, when exactly the expansion began.

\section{Symmetry of energy and information}

Einstein's well-known formula for the energy equivalent of a mass is very similar to a comparable powerful new formula for the information equivalent of time [6.]

$$
\begin{aligned}
& E=m c^{2} \\
& I=t c^{2}
\end{aligned}
$$

The equation (27) was found by me. It is compatible with the definitions of time and information in chapter 1. Any period of time corresponds to a certain amount of information. Since the square of the speed of light is contained in the information according to (27), the information content of one second, for example, is enormous.

\section{The energy-time-symmetry and the entropy of the universe}

While in quantum physics one speaks of an "energy-time-blur", the simple energy-time relationship applies to the mass development of the universe:

$$
m_{\text {uni }} t_{\text {uni }}=\text { const. }
$$


The product of the space mass $m_{u n i}$ and the space age $t_{u n i}$ remains constant [16.]

It is interesting to note that relativistic physics today accepts the speed-dependent increase in mass on the one hand and on the other hand cannot couple it with the relativity of time as misunderstood by Einstein. He postulated a time dilation for inertial systems which is independent of mass. In nature, however, a time dilation of every moving mass in relation to its rest mass occurs. It is therefore impossible for 2 observers with clocks on one inertial system each to have their clocks follow each other. Thus equation (28) applies to every moving mass.

Thus the following symmetry must apply between the rest state $m_{0}, t_{0}$ and the moving state $m_{b}, t_{b}$ of a mass with $v$ [17.]:

$$
m_{0} t_{0}=m_{b} t_{b}=\frac{m_{0}}{\sqrt{1-\frac{v^{2}}{c^{2}}}} \sqrt{1-\frac{v^{2}}{c^{2}}} t_{0}
$$

Thereby $t_{0}$ is an arbitrary period of time, which changes to $t_{b}$ in the moved state.

If two masses fall freely from rest until they collide, equation (29) then also applies to each of the two masses.

Equation (29) already shows the constant entropy $S_{0}=S_{b}=\frac{k}{\hbar} m_{0} t_{0} c^{2}=$ const. for gravity, which also applies to space as a whole [18.] (see also (25)).

$$
S_{\text {uni }}=\frac{k}{\hbar} m_{\text {b uni }} t_{v \text { uni }} c^{2}=\frac{k}{\hbar} m_{d \text { uni }} t_{\text {z uni }} c^{2}=\text { const. }
$$

\section{Symmetry of energy and information conservation}

The gravitational energy of the universe $E_{\text {grav uni }}$ corresponds exactly to the energy equivalent of the mass $E_{m \text { uni }}$ contained in the universe. While the gravitational energy is negative, the mass energy is positive. The mass decays and its positive energy becomes smaller.

At the same time, the gravitational energy increases because it becomes less negative. Its amount, however, becomes smaller just like the mass energy [19.].

$$
\begin{aligned}
& \sum E_{\text {grav uni }}+\sum E_{\text {muni }}=0 \\
& -\frac{\gamma m_{u n i} i^{2}}{r_{u n i}}+m_{u n i} c^{2}=0
\end{aligned}
$$

This leads together with the corrugation radius $r_{u n i}$ to the calculation possibility of the space mass with the space age $r_{u n i}=t_{\text {uni }} c$ :

$$
-\frac{\gamma m_{u n i}}{r_{u n i}}+c^{2}=0 \quad m_{u n i}=\frac{r_{u n i} c^{2}}{\gamma}=\frac{t_{u n i} c^{3}}{\gamma}
$$

The space mass is therefore $m_{u n i}=1.75710^{53} \mathrm{~kg}$.

What does the equation for information, which is symmetrical to equation (31), look like? The sum of current past information $I_{v}$ uni and future information $I_{z}$ uni , which is still contained in matter as structural information, is constant.

$$
I_{v \text { uni }}+\left|I_{z \text { uni }}\right|=\text { const. }
$$

With equation (27), therefore, the sum of past $t_{v \text { uni }}$ and the amount of the future $t_{z \text { uni }}$ :

$$
t_{v \text { uni }}+\left|t_{\text {zuni }}\right|=\text { const. }
$$

While in the expansion of the universe the past information is constantly growing, the amount of future information is decreasing.

\section{Symmetry of gravitationally moving and decayed masses}

The free fall from rest until the collision of two masses results in a speed-related increase in mass $\Delta m_{\text {grav }}$ of both masses. Furthermore, the universal mass decay $\Delta m_{m z}$ of both masses in a certain time is valid. 
The connection between the decrease in mass due to mass decay and the speed-related increase in mass due to gravitation can be specified for a two-mass system as follows:

$$
\frac{\Delta m_{1 m z}}{\Delta m_{2 m z}}=\frac{\Delta m_{2 \text { grav }}}{\Delta m_{1} \text { grav }}=\frac{m_{1}}{m_{2}}
$$

The equation (36) derived in Quelle [20.] was checked with sample calculations for various two-mass systems.

\section{Symmetry of time dilations in the two-mass system}

\subsection{Translation}

Since at least two masses are required for gravity to work, there must also be two time dilations, one on the first and one on the second mass.

The importance of this viewpoint can be seen from Newton's gravitational equation, which is derived below. At the distance $r$ of both masses, mass 1 experiences the time dilation $\Delta t_{1} / t_{1 b}$ on its surface by mass 2 .

Mass 2 in turn experiences the time dilation $\Delta t_{2} / t_{2 b}$ on its surface by mass 1 . The time span $t_{b}$ is the time span which is usually only slightly changed compared to the time span $t_{0}$ due to gravity. For most calculations, therefore, the time dilation is approximate: $\Delta t / t_{b}=\Delta t / t_{0}$.

For the specific time dilation $\Delta t / t_{b}$ can be written [21.]:

$$
\frac{\Delta t_{1}}{t_{1 b}}=-\frac{\gamma m_{2}{ }^{2}}{c^{2} r_{b}\left(m_{1}+m_{2}\right)} \quad \frac{\Delta t_{2}}{t_{2 b}}=-\frac{\gamma m_{1}{ }^{2}}{c^{2} r_{b}\left(m_{1}+m_{2}\right)}
$$

with $\frac{1}{r_{b}}=\frac{1}{r_{1}+r_{2}}-\frac{1}{r}=\frac{h}{r(r-h)}\left(\right.$ spherical masses with the radii $\left.r_{1}, r_{2}\right)$

The connection according to equation (37) was compared and verified with the following important experiments of the past to prove time dilation and to prove the correctness of both theories of relativity:

1. "Pound/Rebka"; 2. "Hafele/Keating"; 3. "Maryland"; 4. "Briatore et al."; 5. "Chou et al. "

On average, the calculation results according to (37) come somewhat closer to the experimental data than the predictions for the time dilations of the two theories of relativity.

\subsection{Rotation}

Of course there must also be symmetrical time dilations in rotating two-mass systems according to equation (37). Each mass influences the time flow on the other mass [22.]:

$$
\frac{\Delta t_{1}}{t_{1 b}}=-\frac{\gamma m_{2}^{2}}{2 c^{2} r\left(m_{1}+m_{2}\right)} \quad \frac{\Delta t_{2}}{t_{2 b}}=-\frac{\gamma m_{1}{ }^{2}}{2 c^{2} r\left(m_{1}+m_{2}\right)}
$$

In equation (38) $r$ is the centre distance of both masses. Why the factor 2 must be in the denominator for the time dilation of the rotation in relation to the translation according to (37), becomes clear in the next chapter.

\section{Symmetry of time dilation and gravitation in the two-mass system}

The relationship between time dilation and gravitational energy in a two-mass system is fundamental to understanding gravity [17.]. With equation (29) and a general mass and time change in form:

$$
\Delta m=m_{b}-m_{0} \quad \Delta t=t_{b}-t_{0}
$$

can be written immediately:

$$
\frac{\Delta m}{m_{0}}=\frac{\Delta m}{m}=-\frac{\Delta t}{t_{b}}
$$

\subsection{Translation}

Two masses should be at rest and at a certain distance from each other and then fall upon each other. The equality of potential and kinetic energy applies. The potential energy results from the integration of the gravitational force from the distance $r$ until their surfaces touch. Thus the $r_{b}$ in equation (37) is created:

$$
E_{\text {grav }}=E_{\text {kin } 1}+E_{\text {kin } 2} \approx \frac{m_{1}}{2} v_{1}^{2}+\frac{m_{2}}{2} v_{2}^{2}
$$

However, the kinetic energy must be used in a precise relativistic notation: 


$$
\begin{aligned}
\gamma \frac{m_{1} m_{2}}{r_{b}} & =\left(\frac{m_{1}}{\sqrt{1-\frac{v_{1}^{2}}{c^{2}}}}-m_{1}\right) c^{2}+\left(\frac{m_{2}}{\sqrt{1-\frac{v_{2}^{2}}{c^{2}}}}-m_{2}\right) c^{2} \\
\gamma \frac{m_{1} m_{2}}{r_{b}} & =\left(m_{1 b}-m_{1}\right) c^{2}+\left(m_{1 b}-m_{1}\right) c^{2} \\
\gamma \frac{m_{1} m_{2}}{r_{b}} & =\Delta m_{1} c^{2}+\Delta m_{2} c^{2}
\end{aligned}
$$

With equation (40) you can write for (44):

$$
\gamma \frac{m_{1} m_{2}}{r_{b}}=-\frac{\Delta t_{1}}{t_{1 b}} m_{1} c^{2}-\frac{\Delta t_{2}}{t_{2 b}} m_{2} c^{2}
$$

Now the time dilations according to equations (37) can be inserted into (45):

$$
\gamma \frac{m_{1} m_{2}}{r_{b}}=\frac{\gamma m_{2}^{2}}{c^{2} r_{b}\left(m_{1}+m_{2}\right)} m_{1} c^{2}+\frac{\gamma m_{1}^{2}}{c^{2} r_{b}\left(m_{1}+m_{2}\right)} m_{2} c^{2}
$$

The equation can be simplified by excluding gravitational energy:

$$
\begin{aligned}
& \gamma \frac{m_{1} m_{2}}{r_{b}}=\gamma \frac{m_{1} m_{2}}{r_{b}}\left(\frac{m_{2}}{m_{1}+m_{2}}+\frac{m_{1}}{m_{1}+m_{2}}\right) \\
& \gamma \frac{m_{1} m_{2}}{r_{b}}=\gamma \frac{m_{1} m_{2}}{r_{b}}
\end{aligned}
$$

In order to obtain the Newtonian gravitational energy of two masses falling on each other from rest from time dilation, both time dilations are therefore absolutely necessary, which can be calculated according to equation (37).

\subsection{Rotation}

Two masses should rotate around a common centre of gravity. The frequent double stars or the Earth-Moon system are suitable examples. In the rotating two-mass system, the amounts of gravitational and centripetal force are equal:

$$
F_{\text {grav }}=F_{z p 1}+F_{z p 2}
$$

As is well known, the circumferential speeds $v_{1}$ and $v_{2}$ can be used for this purpose:

$$
\gamma \frac{m_{1} m_{2}}{r^{2}}=\frac{m_{1} v_{1}^{2}}{r}+\frac{m_{2} v_{2}^{2}}{r}
$$

By multiplication with $r$, an expression with the gravitational energy and double the kinetic energy is obtained:

$$
\gamma \frac{m_{1} m_{2}}{r}=m_{1} v_{1}^{2}+m_{2} v_{2}^{2}
$$

Comparable to equation (42), it is now possible to write with the inclusion of relativistic kinetic energy:

$$
\gamma \frac{m_{1} m_{2}}{r}=2\left(\frac{m_{1}}{\sqrt{1-\frac{v_{1}^{2}}{c^{2}}}}-m_{1}\right) c^{2}+2\left(\frac{m_{2}}{\sqrt{1-\frac{v_{2}^{2}}{c^{2}}}}-m_{2}\right) c^{2}
$$

According to equations (43) and (44) can be written:

$$
\gamma \frac{m_{1} m_{2}}{r}=2 \Delta m_{1} c^{2}+2 \Delta m_{2} c^{2}
$$

In equation (53) the two time dilations can be introduced again, this time according to equation (38):

$$
\begin{aligned}
& \gamma \frac{m_{1} m_{2}}{r}=-2 \frac{\Delta t_{1}}{t_{1 b}} m_{1} c^{2}-2 \frac{\Delta t_{2}}{t_{2 b}} m_{2} c^{2} \\
& \gamma \frac{m_{1} m_{2}}{r}=2 \frac{\gamma m_{2}{ }^{2}}{2 c^{2} r\left(m_{1}+m_{2}\right)} m_{1} c^{2}+2 \frac{\gamma m_{1}{ }^{2}}{2 c^{2} r\left(m_{1}+m_{2}\right)} m_{2} c^{2}
\end{aligned}
$$

The left side of the equation for gravitational energy can also be simplified for rotation:

$$
\gamma \frac{m_{1} m_{2}}{r}=\gamma \frac{m_{1} m_{2}}{r}
$$


This means that in the rotating two-mass system time dilation occurs in the calculation form given in equation (38).

\section{Symmetry of fall, circulation and oscillation speeds in the two-mass system}

With the relativistic relationship $\frac{1}{\sqrt{1-\frac{v^{2}}{c^{2}}}}-1=\frac{\Delta m}{m}$ and the time dilations according to equations (37) and (38), it is very easy to derive relativistic speed equations for two-mass systems that stand up to any scrutiny [23].

\subsection{Translation}

$$
\begin{aligned}
& v_{1}=c \sqrt{1-\frac{1}{\left[1-\frac{\Delta t_{1}}{t_{1 b}}\right]^{2}}}=c \sqrt{1-\frac{1}{\left[1+\frac{\gamma m_{2}^{2}}{c^{2} r_{b}\left(m_{1}+m_{2}\right)}\right]^{2}}} \\
& v_{2}=c \sqrt{1-\frac{1}{\left[1-\frac{\Delta t_{2}}{t_{2 b}}\right]^{2}}}=c \sqrt{1-\frac{1}{\left[1+\frac{\gamma m_{1}^{2}}{c^{2} r_{b}\left(m_{1}+m_{2}\right)}\right]^{2}}}
\end{aligned}
$$

\subsection{Rotation}

$$
\begin{aligned}
& v_{1}=c \sqrt{1-\frac{1}{\left[1-\frac{\Delta t_{1}}{t_{1 b}}\right]^{2}}}=c \sqrt{1-\frac{1}{\left[1+\frac{\gamma m_{2}^{2}}{2 c^{2} r\left(m_{1}+m_{2}\right)}\right]^{2}}} \\
& v_{2}=c \sqrt{1-\frac{1}{\left[1-\frac{\Delta t_{2}}{t_{2 b}}\right]^{2}}}=c \sqrt{1-\frac{1}{\left[1+\frac{\gamma m_{1}^{2}}{2 c^{2} r\left(m_{1}+m_{2}\right)}\right]^{2}}}
\end{aligned}
$$
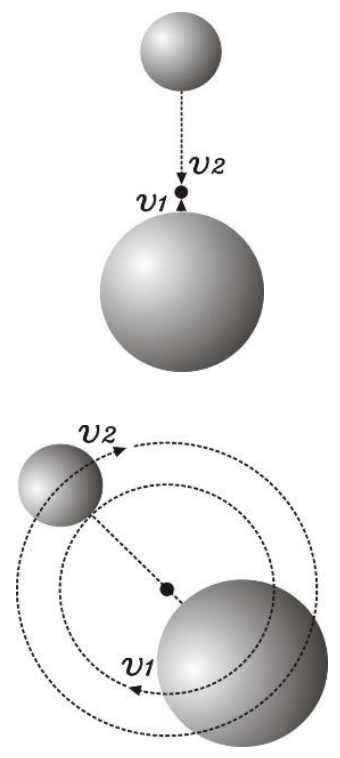

12.3 Quantum system (hydrogen atom according to the Bohr atom model)

$$
\begin{aligned}
& v_{1}=c \sqrt{1-\frac{1}{\left[1+\frac{\gamma m_{2}^{2}}{2 c^{2} r\left(m_{1}+m_{2}\right)} \frac{F_{e}}{F_{\text {grav }}}\right]^{2}}} \\
& v_{2}=c \sqrt{1-\frac{1}{\left[1+\frac{\gamma m_{1}^{2}}{2 c^{2} r\left(m_{1}+m_{2}\right)} \frac{F_{e}}{F_{\text {grav }}}\right]^{2}}}
\end{aligned}
$$

At the speeds of electron and proton in the hydrogen atom, the ratio of the electric force to the gravitational force must be taken into account.

\section{Symmetry of fall and rotation times in the two-mass system}

\subsection{Translation}

In order to determine the fall time of two masses from rest to collision in a two-mass system, the wellknown non-linear differential equation of the second order must be solved:

$$
\frac{d^{2} r}{d t^{2}}=-\gamma \frac{m_{1}+m_{2}}{r^{2}}
$$

This equation cannot be solved analytically, but can be solved numerically for known object sizes.

For the fall time of two masses, which fall on each other from the rest, the centre distance $r$ and the surface distance $h$, I have given the following formula for the first time [24.]:

$$
t_{\text {fall }}=\sqrt{\frac{2 h r^{2}}{\gamma\left(m_{1}+m_{2}\right)}}
$$

With the simplifications of a constant acceleration $g$ and neglecting the size of the falling mass $m_{2}$, one quickly arrives at the well-known formula $t_{\text {fall }}=\sqrt{2 \mathrm{~h} / \mathrm{g}}$ from this formula. 


\subsection{Rotation}

The well-known formula for the period of rotation of two masses around a common centre of gravity is very similar to equation (64):

$$
T=2 \pi \sqrt{\frac{r^{3}}{\gamma\left(m_{1}+m_{2}\right)}}
$$

Equation (64) changes to formula (65) for 2 masses, whose radii $r_{1}$ and $r_{2}$ are small compared to the centre distance $(h \approx r)$, except for the factor 2 under the root.

\section{Summary}

The ideas and calculations contained here have been developed in more than 13 years of theoretical research and, where possible, tested. Above all, dual symmetries are revealed to the reader here, which are firmly anchored in the structure of nature.

"Mathematics is the instrument that mediates between theory and practice, between thinking and observation: It builds the connecting bridge and makes it increasingly sustainable. It is for this reason that our entire contemporary culture, insofar as it is based on the spiritual penetration and service of nature, finds its foundation in mathematics." [25.].

All equations have been verified with concrete calculations. The reader is invited to make his own calculations. For only in this way will the equations found unfold their effect and ultimately contribute to the understanding of nature.

\section{References}

[1.] Hardy, Godfrey Harold, A Mathematican's Apology, University Press, Cambridge 1940, ISBN 978-0-521-42706-7.

[2.] Weizsäcker, Carl Friedrich von, Die Einheit der Natur, Deutscher Taschenbuch Verlag GmbH \& Co. KG, München, 1971.

[3.] Wiener, Norbert, Cybernetics or control and communication in the animal and the machine, New York / London, 1961.

[4.] Callenger, Craig, Ist Zeit eine Illusion, Spektrum der Wissenschaft, Oktober 2010, SdW-Verlag

[5.] Gimsa, Andreas, The beauty of nature, 2nd edition, p.5, Gieselmann Druck- und Medienhaus, Potsdam, 2014, ISBN 978-3-923830-94-7.

[6.] See Source [5.], p.31.

[7.] Gimsa, Andreas, The metric of space-time, 2nd edition, p.8, Books on Demand GmbH, Norderstedt, 2020, ISBN 978-3-00-064784-0.

[8.] See Source [7.], p.46-52.

[9.] See Source [7.], p.17-25.

[10.] See Source [7.], p.21.

[11.] Meschede, Dieter, Gerthsen Physik, 25. Auflage, S. 981, Springer-Verlag Heidelberg, 2015, ISBN 978-3-662-45977-5.

[12.] Gimsa, Andreas, Der Wellenwiderstand des Vakuums, Teil1, p.3-5, Kuss GmbH, Potsdam, 2015, ISBN 978-3-00-051924-6.

[13.] Dirac, P.A.M, Proc. Roy. Soc. A 133 60, 1931.

[14.] See Source [7.], p.59.

[15.] See Source [7.], p.42.

[16.] See Source [5.], p.42.

[17.] See Source [5.], p.6.

[18.] See Source [7.], p.26.

[19.] See Source [7.], p.78.

[20.] See Source [7.], p.54.

[21.] See Source [5.], p.13.

[22.] Gimsa, Andreas, Dual mass systems under the influence of gravitation, p.23, Books on Demand GmbH, Germany 2017, ISBN 978-3-00-057218-0.

[23.] See Source [22.] p.13-24.

[24.] See Source [7.], p.52. 
[25.] Hilbert, David, Naturerkennen und Logik, Königsberg, 1930 Versammlung Deutscher Naturforscher und Ärzte; Gesammelte Abhandlungen, Dritter Band, S.385, Verlag von Julius Springer, Berlin, 1935. 